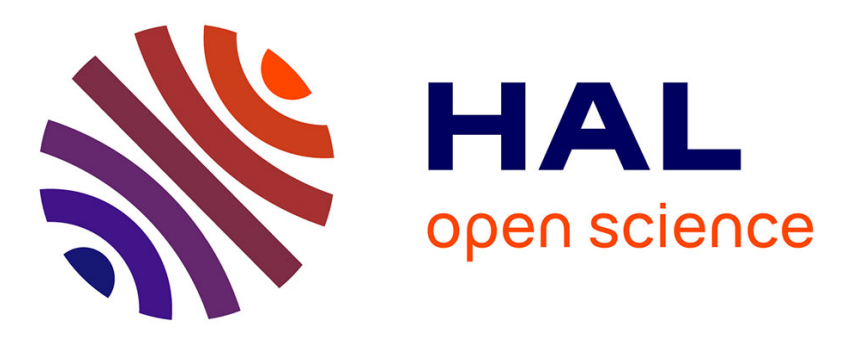

\title{
Statistical-mechanical approach to study the hydrodynamic stability of the stably stratified atmospheric boundary layer
}

Guillaume Nevo, Nikki Vercauteren, Amandine Kaiser, Bérengère Dubrulle, Davide Faranda

\section{To cite this version:}

Guillaume Nevo, Nikki Vercauteren, Amandine Kaiser, Bérengère Dubrulle, Davide Faranda. Statistical-mechanical approach to study the hydrodynamic stability of the stably stratified atmospheric boundary layer. Physical Review Fluids, 2017, 2 (084603), 10.1103/PhysRevFluids.2.084603 . hal-01413073v2

\section{HAL Id: hal-01413073 \\ https://hal.science/hal-01413073v2}

Submitted on 6 Jul 2017

HAL is a multi-disciplinary open access archive for the deposit and dissemination of scientific research documents, whether they are published or not. The documents may come from teaching and research institutions in France or abroad, or from public or private research centers.
L'archive ouverte pluridisciplinaire HAL, est destinée au dépôt et à la diffusion de documents scientifiques de niveau recherche, publiés ou non, émanant des établissements d'enseignement et de recherche français ou étrangers, des laboratoires publics ou privés. 


\title{
A statistical-mechanical approach to study the hydrodynamic stability of stably stratified atmospheric boundary layer
}

\author{
G. Nevo \\ SPEC, CEA, CNRS, Université Paris-Saclay, \\ CEA Saclay 91191 Gif-sur-Yvette, France \\ ICFP, Physics Department, \\ École Normale Supérieure, PSL Research University, \\ 24, rue Lhomond, 75005 Paris
}

N. Vercauteren, A. Kaiser

FB Mathematik und Informatik, Freie Universität Berlin, Arnimallee 6, 14195 Berlin, Germany

B. Dubrulle

SPEC, CEA, CNRS, Université Paris-Saclay, CEA Saclay 91191 Gif-sur-Yvette, France

D. Faranda*

LSCE-IPSL, CEA Saclay l'Orme des Merisiers, CNRS UMR 8212 CEA-CNRS-UVSQ,

Université Paris-Saclay, 91191 Gif-sur-Yvette, France

\begin{abstract}
We study the hydrodynamic equilibrium properties of the stably stratified atmospheric boundary layer from measurements obtained in the Snow-Horizontal Array Turbulence Study (SnoHATS) campaign at the Plaine Morte Glacier in the Swiss Alps. Our approach is based on a combination of dynamical systems techniques and statistical analysis. The main idea is to measure the deviations from the behavior expected by a turbulent observable when it is close to a transition between different metastable states. We first assess the performance of our method on the Lorenz attractor, then on a turbulent flow. The results show that the method recognizes subtle differences among different stable boundary layers turbulence regimes and may be used to help characterise their transitions.
\end{abstract}

\section{INTRODUCTION}

Mixing in the atmospheric boundary layer (ABL) strongly depends on the properties of the surface, on the dynamics induced by atmospheric motions as well as on thermodynamic and radiative effects. All those ingredients complicate the representation of the lower tropospheric dynamics in climate models, introducing an important source of inaccuracy in weather forecast and climate simulations [1-3]. ABL parameteriziations are usually based on the reduction of the Navier-Stokes equations to some simpler relations. They are obtained by lumping the subgrid contributions to empirical control parameters. The order of the turbulence closure and the use of local or nonlocal mixing approaches are the two main points faced in the choice of a ABL parameterization scheme. Particularly difficult is the choice of parameterization of the stably stratified boundary layer [4]. Indeed it is known that numerical weather prediction or climate models show a great sensitivity to the model mixing formulations in stable boundary layers (SBL) situations, which occur frequently at nighttime or in Polar Regions. However, progress in understanding and representing the SBL is slow (see [5] and references therein).

Based on observational evidence, the SBL is often broadly classified in weakly stable and strongly stable regimes. Idealised physical models have also identified different regimes, in particular a quasi-steady turbulent regime and an intermittent regime [6]. The intermittency of turbulence in the strongly stable situations has been shown to be at least in part due to shear instabilities resulting from localised wind accelerations; these accelerations can be the results of internal gravity waves, density currents, wind gusts or other motions of unknown origin [7 [8]. Such non-turbulent, small-scale motions are usually denoted as submeso motions and can take various forms [9]. With sufficiently weak large-scale flow, i.e. when the mean wind speed is small, the non-turbulent part of the flow can consist primarily of these highly non-stationary submeso, which scales can vary from a few meters to a few kilometers [10] [11. In this context, the turbulence cannot maintain equilibrium with the constantly changing wind speed and direction, making the flow dynamically unstable (despite a thermally strongly stable stratification). This lack of equilibrium can prevent the formation of an inertial subrange, and makes the use of classical turbulence parameterizations based on the mean wind speed and stratification unphysical. Specifically, the lack of equilibrium could trigger a need for higher order turbulence closure schemes. We stress that in this work, wherever not specified, we refer to stability as the dynamic 
stability.

Recent work analysing SBL dynamics from field measurements has focused on detecting periods of non-stationary turbulence and how it relates to submeso motions [9, 11-13. Overall, recent contributions support the need of taking a regime approach to parameterizing the SBL [7, 12, 14, 15. Monahan et al. [14 further found a correspondence between two statistically classified SBL flow states and the dynamically unstable and stable branches of the equilibrium curves of the idealized Couette flow model suggested by van de Wiel [16]. Despite being unstable to pertubations in the model, roughly half of the observed states fell along the unstable equilibrium branch. This motivates further analyses of dynamical stability properties of field data. Starting from the measurements of the wind vector fields and/or temperature, our goal is then to define in an unambiguous way the dynamical stability of the observed regimes in the SBL. Indeed, identified regimes can be interpreted as metastable states in dynamical systems. Since the SBL response varies as a function of the atmospheric and land surface processes and can be strongly impacted by non-stationary submeso motions, the task of assessing the dynamical stability of the different (metastable) states is extremely complex and needs ad-hoc tools.

Here we propose to determine the dynamical stability of the SBL by using a combination of dynamical systems concepts and stochastic processes tools. In chaotic or turbulent systems, the dynamics consists of a complex trajectory in the phase space. The ensemble of the states visited is generally a compact object called the attractor. In the attractor, the trajectory spends most of the time around metastable points making sporadic jumps among them. When the attractor cannot be reconstructed, one must rely on a good observable, capable to project the complex behavior of the system in just one (a few?) dimension. When the system is close to a metastable state, the dynamics appear like a Langevin equation i.e. it consists of noisy fluctuations around a well define value of the observable. When the system follows unstable orbits, the dynamics is more irregular and different time scale appears in the signal. By using the auto-regressive moving-average (ARMA) processes, we have defined a [0,1] distance from the basic Langevin behavior. Here we show that, by slicing a signal and applying such indicators on the subsets of the original time series, we can assess the stability properties of the underlying systems.

Before applying the indicator to atmospheric measurements, we test our indicator in two systems of increasing complexity: the Lorenz [17] flow, a chaotic system consisting of three differential equations, and the von Kármán flow, a laboratory turbulent flow that possesses a low dimensional stochastic attractor. We chose those systems for different reasons: the Lorenz attractor is a well known but non-trivial object in dynamical systems theory and features three unstable fixed points with different degrees of instability. The von Kármán experiment features non-trivial metastable states and we have been able to reconstruct its attractor using as control parameter $\gamma$, a function of the torque applied to the flow. Once the method has been validated on these systems, we apply it to data from atmospheric turbulence obtained during the Snow-Horizontal Array Turbulence Study (SnoHATS) campaign [18. As a final check of our method, we assume to know - from different sources that will be detailed later - the metastable states analyzed and check the ability of our indicator to discriminate their stability properties.

The paper is organised as follows: in the next section we give an overview of the ARMA modeling and explain how we build our stability indicators. Then we validate our method on the two model systems of increasing complexity. Finally we apply our method to atmospheric data. We discuss the results and their implications for the PBL modeling.

\section{METHODS}

The Autoregressive Moving-average model (ARMA) is a class of linear processes. It has been widely used over the past decades, especially in econometrics and finance to forecast markets trends. We briefly show the normal form of an $\operatorname{ARMA}(p, q)$ model and some criteria used to fit it to a time series (see [19] for a detailed review).

Let us consider a series $X(t)$ of an observable with unknown underlying dynamics. We further assume that for a time scale $\tau$ of interest, the time series $X_{t_{1}}, X_{t_{2}}, \ldots, X_{t \tau}$ represents a stationary phenomenon. Since $X_{t}$ is stationary, we may then model it by an $\operatorname{ARMA}(\mathrm{p}, \mathrm{q})$ process such that for all $t$ :

$$
X_{t}=\sum_{i=1}^{p} \phi_{i} X_{t-i}+\varepsilon_{t}+\sum_{j=1}^{q} \theta_{j} \varepsilon_{t-j}
$$

with $\varepsilon_{t} \sim W N\left(0, \sigma^{2}\right)$ - where $W N$ stands for white noise - and the polynomials $\phi(z)=1-\phi_{1} z_{t-1}-\ldots-\phi_{p} z_{t-p}$ $\theta(z)=1-\theta_{1} z_{t-1}-\ldots-\theta_{q} z_{t-q}$, with $z \in \mathbb{C}$, have no common factors. Notice that, hereinafter, the noise term $\varepsilon_{t}$ is 
assumed to be a white noise. For a general stationary time series, this model is not unique. However there are several standard procedures for selecting the model which fits at best the data. The one we exploit is the Bayesian information criteria [20]. It is based on the Akaike information criteria (AIC) [21] which was designed to be an approximately unbiased estimate of the Kullback-Leibler index of the fitted model relative to the true model. Assuming we know the likelihood estimators $\beta$ and $\sigma^{2}$ of the fitted model thanks to an innovation algorithm, our ARMA model is the one where $p$ and $q$ minimize

$$
\operatorname{AIC}(\beta)=-2 \ln L_{X}\left(\beta, \sigma^{2}\right)+2(p+q+1)
$$

In order to correct the tendency of the AIC to prefer complex models, we use the BIC (Bayesian information criteria) which introduces a penalty for large-order models:

$$
\mathrm{BIC}=(\tau-p-q) \ln \left(\frac{\tau \sigma^{2}}{\tau-p-q}\right)+\tau(1+\ln \sqrt{2 \pi})+(p+q) \ln \left(\left(\sum_{t=1}^{\tau} X_{t}^{2}-\tau \sigma^{2}\right) /(p+q)\right)
$$

Intuitively, $p$ and $q$ are related to memory lag of the process, while the coefficients $\phi_{i}$ and $\theta_{i}$ represent the persistence: the higher their sum (in absolute value), the slower the system is in forgetting its past history, the higher the correlations in the time series. Most of the time ARMA models are used in econometrics fitting the whole time series and trying to forecast the future trend of the variable. This assumes a correlation with the past and provide some significant results for the very near future. Our interest is rather to use ARMA to detect the local stability. The procedure is the same as for fitting the whole time series: after slicing the time series by intervals $\tau$, we obtain a time series $X_{t-\tau}, \ldots, X_{t-1}$. We then fit each $\operatorname{ARMA}(\mathrm{p}, \mathrm{q})$ model until $p \leq p_{\max }$ and $q \leq q_{\max }$ - where $p_{\max }$ and $q_{\max }$ are predefined threshold values - assessing the best one (according to the BIC criterion). We then compute the stability indicator $\Upsilon$ for the system at time $t$ and then move to time $t+1$ to perform the same analysis on the time series $X_{t-\tau+1}, \ldots, X_{t}$. The idea is to obtain slices of the original dynamics that, despite the non-linearity of the underlying system, locally satisfy the requirement of linearity, Gaussianity and independence on the noise realization required when doing ARMA modeling.

\section{An indicator of stability based on the stochastic models}

We define our stability indicator by using a simple example: even for a complex system, the dynamics near a metastable state resembles to that of a stochastic spring (or of a particle in a quadratic potential). The typical equation associated to those system is the Langevin equation:

$$
\frac{\mathrm{d} X(t)}{\mathrm{d} t}=-\frac{k}{m} X(t)+\frac{1}{m} \xi(t)
$$

with $k$ a frictional force (e.g. the Stokes' drag), $m$ the mass of the particle and $\xi$ a noise term modeling random collisions the particle undergoes. The discretized equation then becomes:

$$
X_{t}=\phi X_{t-1}+\epsilon_{t}
$$

which is an $\operatorname{ARMA}(1,0)$.

Instead, when the system is close to an unstable point, separating multiple basins of attraction, the behavior cannot be described by a Langevin equation as the underlying potential is not quadratic anymore. The change in the shape of the potential introduces new correlation in the time series resulting in higher order ARMA terms. The indicator is then defined as:

$$
\Upsilon(p, q ; \tau)=1-\exp \frac{|\mathrm{BIC}(p, q)-\mathrm{BIC}(1,0)|}{\tau}
$$

In order to simplify notation, from hereinafter we will drop the $\Upsilon$ dependence on $p, q ; \tau$. Thus, $\Upsilon$ gives us a normalized distance between the stablest state the particle could be in $(\Upsilon=0)$ and the state where it really is. The limit $\Upsilon \longrightarrow 1$ correspond to a very unstable state, where the particle is a the edge of a basin of attraction and the probability to jump to another connected basin is high. This is the limit of strong nonlinearities and indicates also that the sub-series cannot be modeled with an ARMA process.

The only free parameter is the choice of $\tau$. To understand its role, we revert to the spring example: the characteristic 
time scale of the problem is the relaxation time of the particle to the basin of attraction. This define the typical time scale of the system. The $\Delta t$ between subsequent observation of the time series should be close to this quantity. Instead, $\tau$ must be a multiple of this quantity but should be smaller than the residence time in the basin of attraction. In previous works, some of the authors of this paper have shown the validity of the $\Upsilon$ indicator to study financial [22] and climate time series 23 .

\section{VALIDATION}

We applied our ARMA method on three different systems: the Lorenz 17 attractor, the data of a von Karman flow experiment and the atmospheric Snow-Horizontal Array Turbulence Study (SnoHATS) dataset that was collected over Plaine Morte Glacier in the Swiss Alps.

\section{Tests on low dimensional dynamical systems}

We begin the analysis by testing the behavior of $\Upsilon$ on the Lorenz [17] system. This system consists of three differential equation and it is a conceptual model of atmospheric convection. The system is:

$$
\dot{x}=\sigma(y-x) \quad \dot{y}=r x-y-x z \quad \dot{z}=x y-b z
$$

$x, y, z$ variables represent respectively the convection strength, the difference of temperature between the surface and the top of the troposphere, and the asymmetry of the convection cells. The parameters $\sigma, r$ are the Prandtl and the Rayleigh numbers, while $b$ is a ratio of critical parameters. The Lorenz attractor (or Lorenz butterfly) shown in figure 1 -top-left consists of 50000 points obtained by iterating the Lorenz equations with $\Delta t=0.1, \sigma=28, r=10, b=8 / 3$. It consists of three unstable fixed points (the centre of the wings and the origin). The evolution of perturbations around fixed points can be quantified by local Lyapunov exponents (Fig. 3a in [24]). In the Lorenz system under consideration, the origin exhibits the largest Lyapunov exponents, hence perturbations will grow the fastest around the origin, making it more unstable than the center of the wings [24]. Moreover, the local dimension of the attractor - a measure the allowed directions to explore locally the phase space - is larger at the origin, making it more unstable (supplementary material of [25]). As observable $X$ we consider the quadratic sum of the variables. We apply the procedure described in the methods section, taking a window $\tau=32 \Delta t$, although the results are insensitive to the choice of $\tau$ providing that $\tau>30$ as we have verified.

The results for $\Upsilon$ are shown in Figure 1-top-right where we pulled back the values of $\Upsilon$ obtained for $X$ to the points of the attractor. The histogram of the results is shown in Figure 1 bottom. The results show that $\Upsilon$ is capable to recognize the location of the unstable fixed points: near the origin the dynamics is very unstable and $\Upsilon$ has higher values, near the center of the wings the dynamics is trapped in the proximity of the unstable fixed points, so that $\Upsilon$ 's values are close to 0 . The low values of $\Upsilon$ obtained on the edges of the attractor are found in correspondence with isolated points of the trajectory and they are possibly due to finite size effects. The histogram shows how complex can be the dynamics of this simple systems of equations: we identify one distinct mode at $\Upsilon=0$ corresponding to the location of the unstable fixed points located at the center of the wings, another two for $0.3<\Upsilon<0.6$ corresponding to the bulk of the statistics with a long tail of values towards $\Upsilon=1$ corresponding to the origin (i.e. the most unstable).

We remark that the same computations can be made with other procedures: for example by determining the local Lyapunov exponents [26] with the requirement of much longer time series, or the stochastic Lyapunov exponents [27] with the requirement of an ensemble of realizations of the same system. It is apparent that $\Upsilon$ allows to obtain the stability with only few observations of the system: in the previous example only 32 at each point of the attractor.

\section{Laboratory turbulence: von Kármán experiment}

We increase the complexity by analyzing the data of a von Kármán swirling flow experiment. This is an important test for the $\Upsilon$ indicator as laboratory turbulent flows are the closest controlled systems with a complex dynamics as the one observed in geophysical motions. The von Kármán flow is generated in a vertical cylinder filled with water by two coaxial, contra-rotating impellers providing energy and momentum flux at the upper and the lower ends of the 

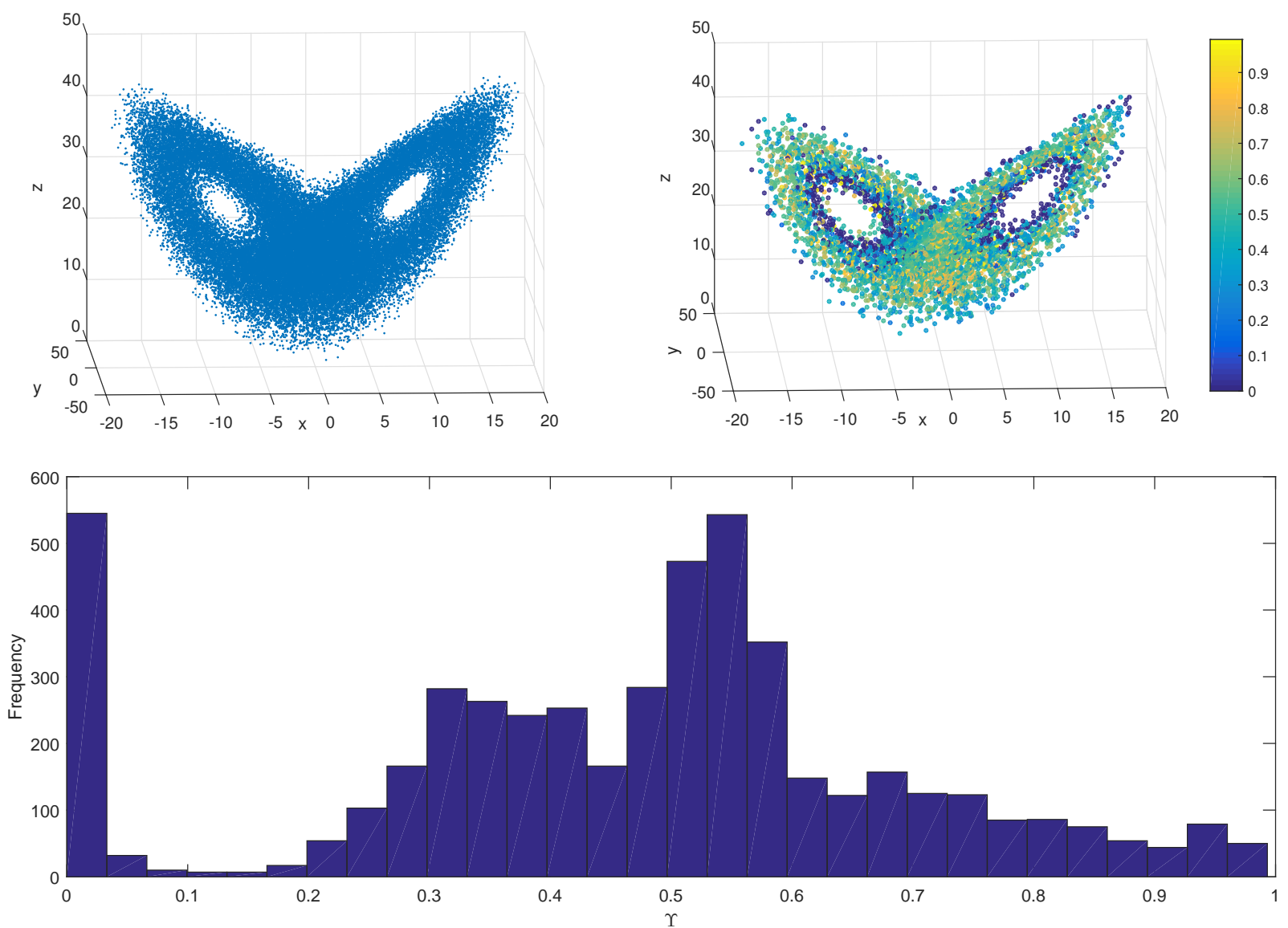

FIG. 1: Top left: Lorenz attractor obtained for 50000 iterations of the Lorenz equations. Top Right: Stability $\Upsilon$ for 4950 points of the attractor. Bottom: histogram of $\Upsilon$ values.

cylinder. The inner radius of the cylinder is $R=100 \mathrm{~mm}$ and the distance between the inner face of the impellers is $H=180 \mathrm{~mm}$, which gives an aspect ratio of $H / R=1.8$. The impellers are driven by two independent motors which can rotate at frequencies up to $10 \mathrm{~Hz}$. The motor frequencies can be either set equal to get exact counter-rotating regime or set to different values $f_{1} \neq f_{2}$. The turbulence properties (anisotropy, fluctuations, and dissipation) are influenced by the geometry of the impellers, i.e., their nondimensional radius $R_{t}$, the oriented angle $\alpha= \pm 72^{\circ}$ between the blades (see Figure 2), the number of blades, and their heights $h_{b}$ [28. "TP87" impellers (with 8 blades) were used during the experiment $[29$.

The experiments analyzed are performed at $R e \sim 10^{5}$ by changing the control parameter $\gamma$ :

$$
\gamma=\frac{C_{1}-C_{2}}{C_{1}+C_{2}}
$$

with $C_{1}$ and $C_{2}$ the torques imposed on the top and the bottom of the cylinder. As $\gamma$ is varied, different dynamical behaviors appear. For $\gamma \sim 0$ the dynamics consists of a fixed point corresponding to a symmetric state where the velocity field consists of four re-circulation cells (Figure 3-left). For $\gamma \neq 0$ the dynamic switches chaotically among the three states of Figure 3 and stochastic chaotic attractors appear.

Such attractors have been obtained in [30] by embedding the reduced impeller rotation frequency $\theta=\left(f_{1}-f_{2}\right) /\left(f_{1}+\right.$ $f_{2}$ ) which, for statistical-mechanics arguments [31, appears as the right observable to track the switching between different metastable states. The 3 dimensional attractor has been obtained in [30] by using the embedding procedure described in Packard et al. 32 and mathematical justified in Sauer et al. 33. We briefly recall the procedure which consists of extracting all partial maxima of $\theta$ time series denoted as Peak $\mathrm{k}_{m}$ from our time series and plot Peak ${ }_{m}$, Peak $_{m+1}$, Peak $_{m+2}$ in a 3-dimensional phase space. Intuitively, as long as the variable is in a metastable state the peaks are just due to noise and they will be close to one another in phase space (top panels of Figure 4). On the opposite case, when the dynamics jumps towards another metastable state, we observe a chaotic attractor (bottom panels). In all the cases, we plot between two consecutive peaks a line colored following the value of $\Upsilon$ we obtained via our ARMA study. The darker the line is, the stronger the fluctuation, which sometimes leads to transitions between 

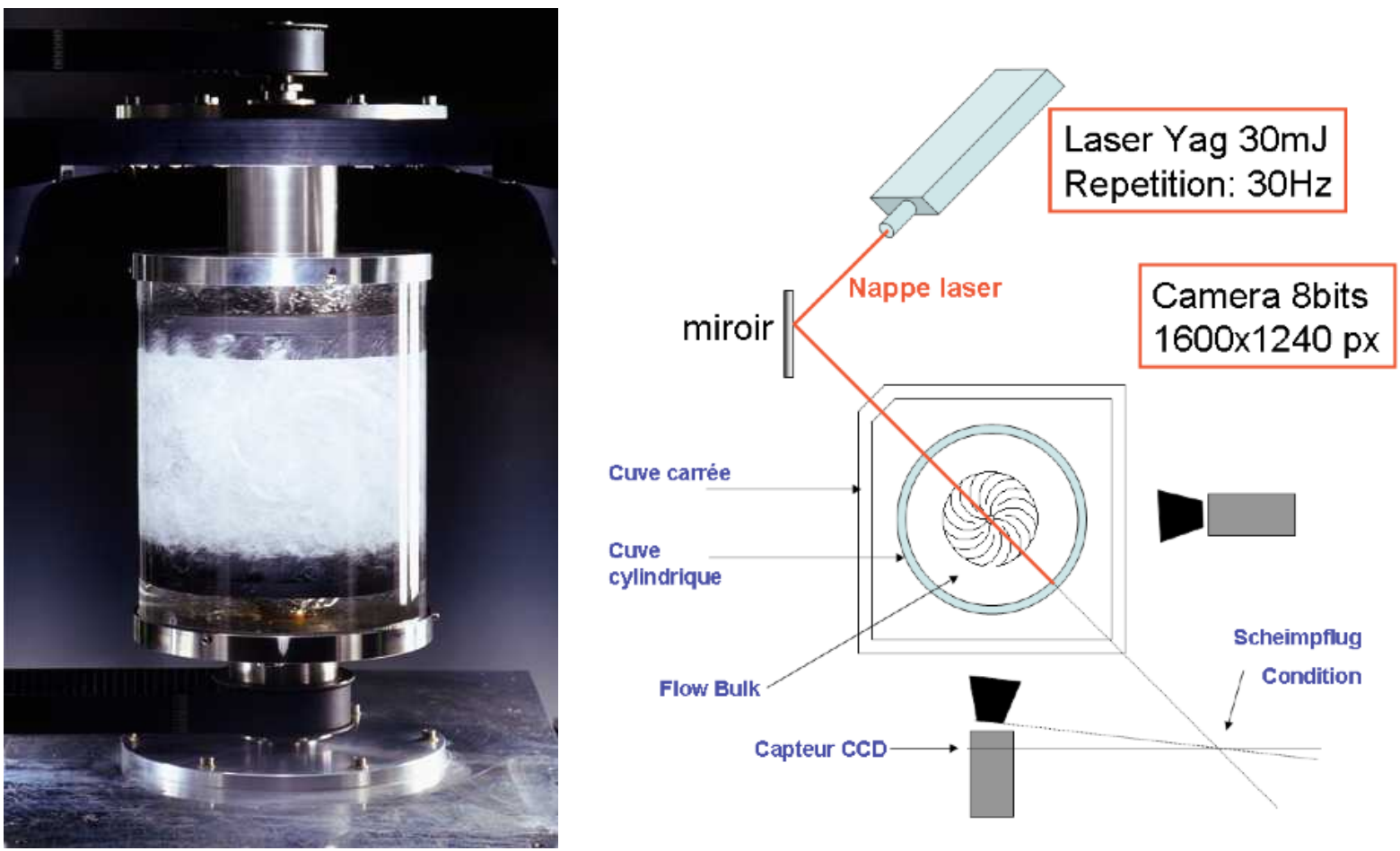

FIG. 2: Setup of the von Kármán experiment. On the left, a picture of the setup designed in CEA Saclay, on the right a scheme of the experiment with the cylinder filled with water in the middle, the laser cutting it by the diagonal to obtain the velocity map and the two sensors on the left and the bottom of the experiment.

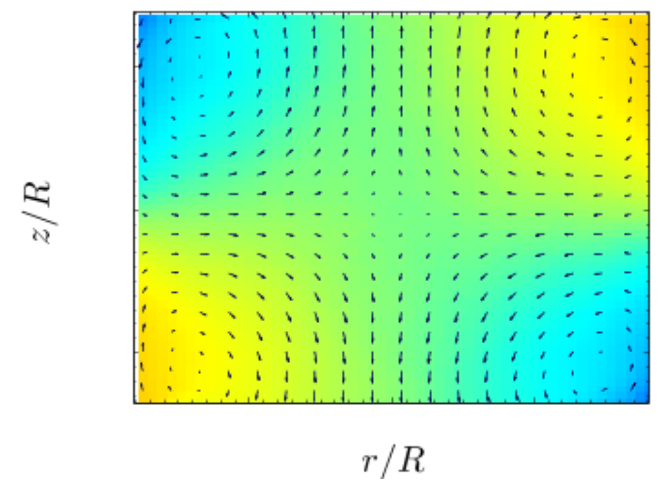

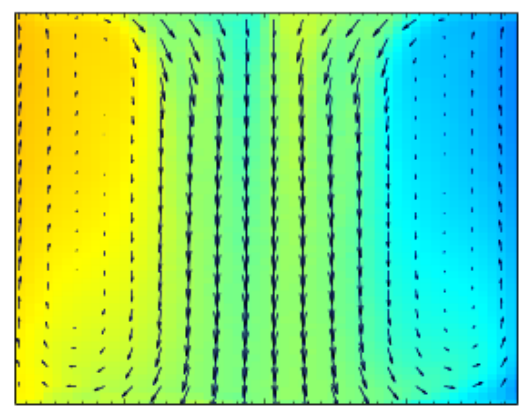

$r / R$

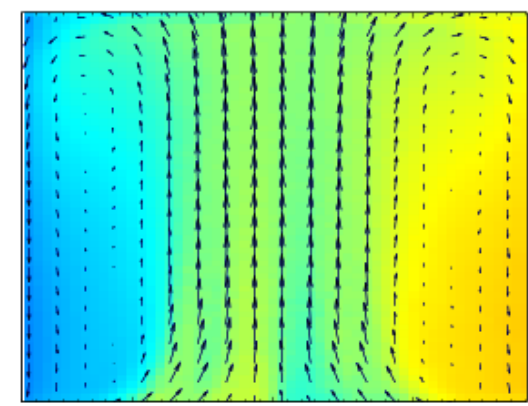

$r / R$

FIG. 3: Steady states of the von Kármán experiment obtained by averaging several instantaneous velocity fields. We can see here three vertical cuts of the cylinder following the setup in Figure 2 at three different times in three different steady states. The velocity map is drawn on each figure with arrows and colors. On the middle and right one we observe two cells counter-rotating (figures are axisymmetric to one another). On the left one we observe the transition state between both with four cells count-rotating.

states. We tested several correlation windows and found that results are stable for $\tau<8$ s. Taking longer time does not allow a good reconstruction of the stability properties as the residence time in the states $\theta>0$ does not generally exceed 10s.

Let us focus on the bottom panels of Figure 4. This attractor consists of three metastable states. These have been detected following an histogram analysis of $f_{1}$ and $f_{2}$ [29] but also based on the dynamical systems analysis performed in [30]. The three metastable states have been also identified by inspection of the velocity fields [34. We find these states back on the attractor: the first corresponding to the symmetric states of coordinates $(0,0,0)$, the second $(0.2,0.2,0.2)$ and the last one in between. In the proximity of these metastable states, low values of $\Upsilon$ indicate that those are stable regions of the attractor. Among the unstable fixed points dark red lines correspond to higher values of $\Upsilon$, i.e. to the unstable paths leading to the transitions between the different states. Furthermore, when we see the dynamic construction of this graphic we observe sorts of preferential transition paths: the stablest state is the bottom one (a state with the two cells contra-rotating). Most of the time the system fluctuates around it then jumps 

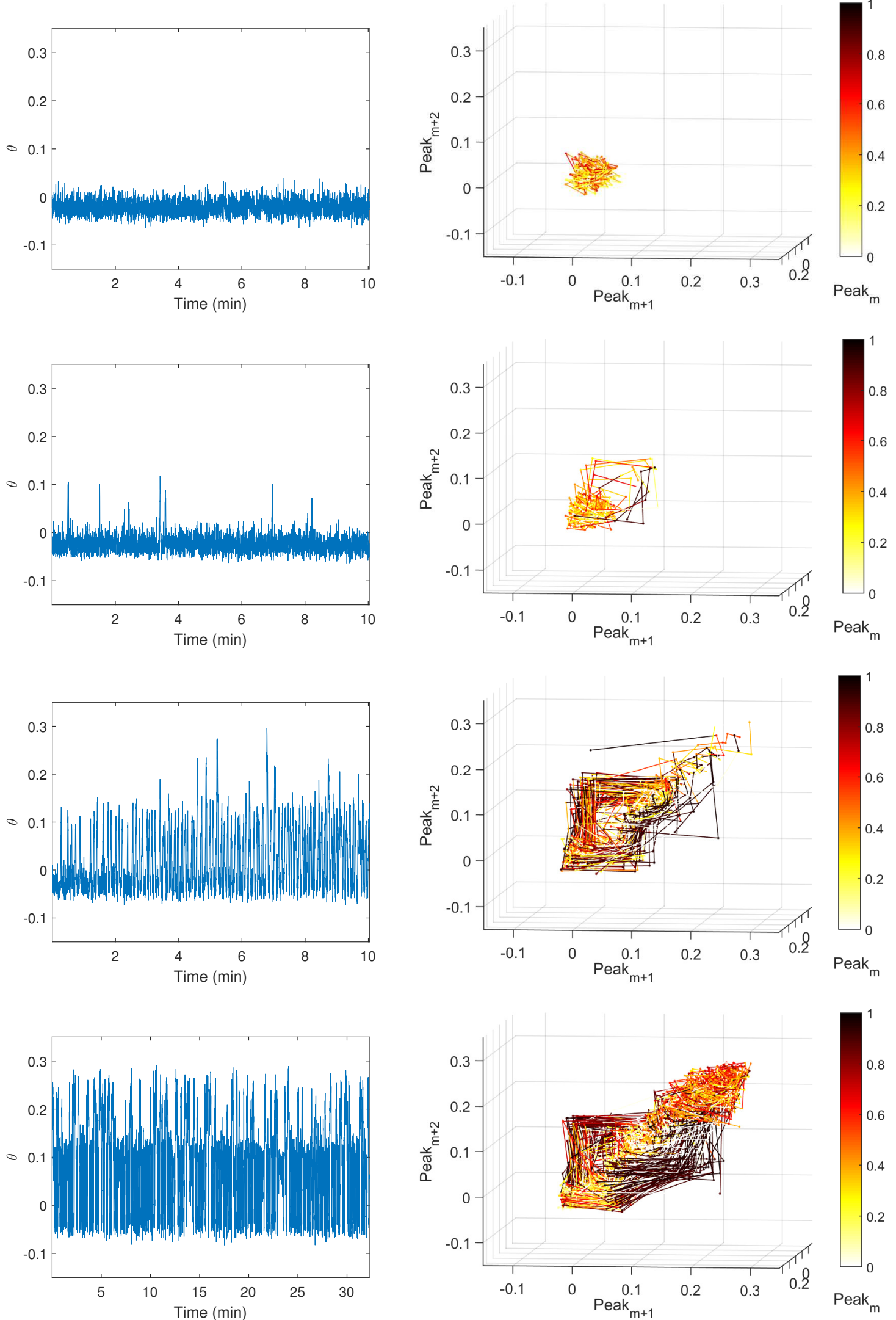

FIG. 4: Left panels: time series of the reduced rotation frequency $\theta$. Right panels: the attractor representation obtained via a three dimensional embedding of $\theta$. From top to bottom $\gamma$ is increased from 0 to 0.06 . The color represents the stability of the points of the attractor measured by $\Upsilon$. 
to the top one (corresponding to the two cells structure in Figure 3). There the dynamics persists for a certain time before going to the third metastable states and almost directly back to the stablest state.

\section{APPLICATION TO ATMOSPHERIC TURBULENCE}

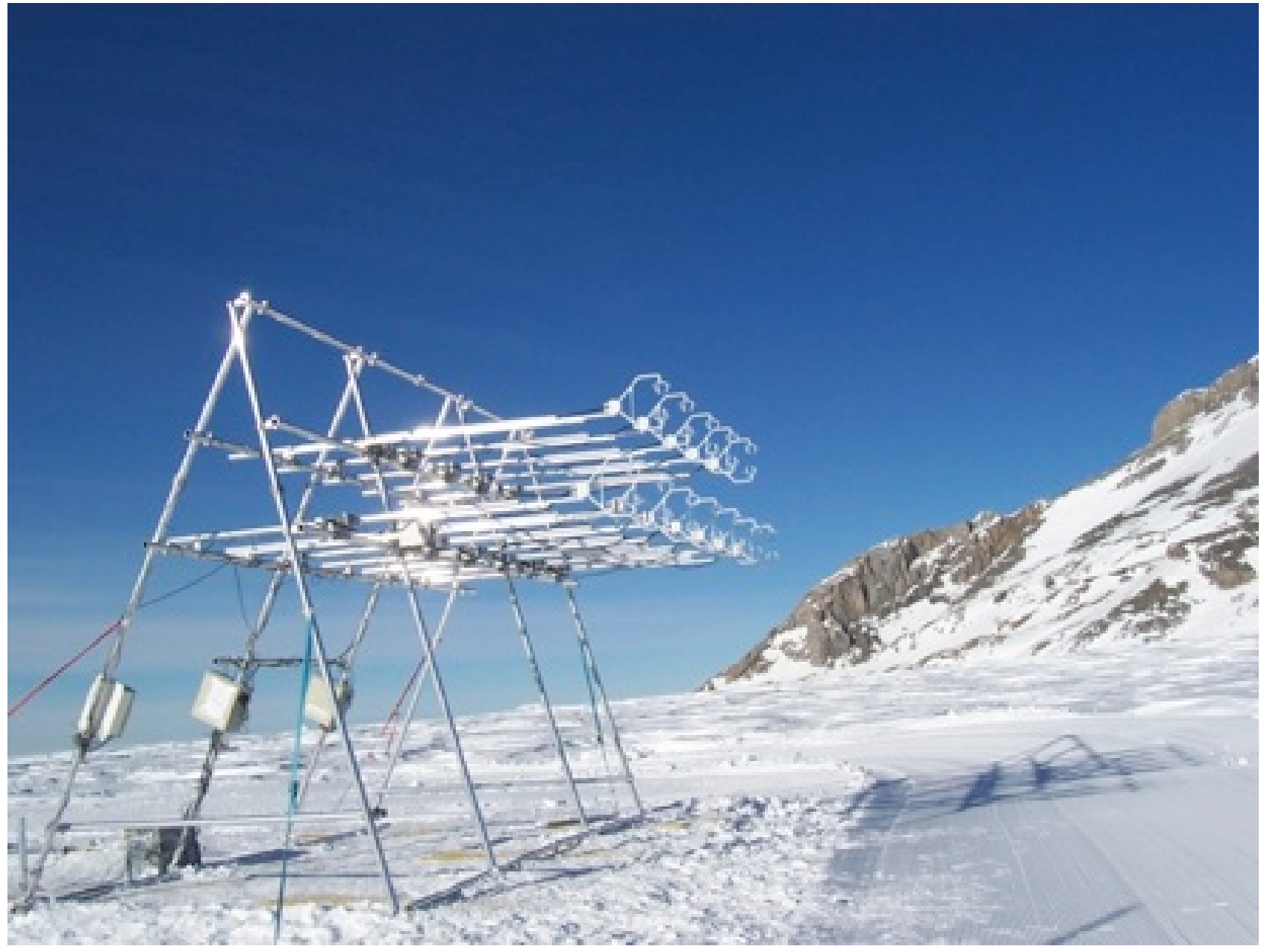

FIG. 5: Experimental setup of the SnoHATS campaign over Plaine Morte Glacier

After the further validation tests on the von Karman flow, we now study experimental atmospheric turbulence data collected near the surface in stably stratified conditions. The analysis is based on the Snow-Horizontal Array Turbulence Study (SnoHATS) dataset that was collected over Plaine Morte Glacier in the Swiss Alps (46.38638N, 7.51788E, 2750-m elevation) from February 2nd to April 19th 2006 [18. The dataset includes three-dimensional measurements of wind velocity recorded at a frequency of $20 \mathrm{~Hz}$ through two vertically separated horizontal arrays of sonic anemometers (see Figure 5), with a total of 12 sonic anemometers (Campbell Scientific, model CSAT3). The height of the two arrays varies during the experiment between 2.82 and $0.62 \mathrm{~m}$ above the snow level depending on snow accumulation. The setup is located over a large flat glacier that provided long periods of stable temperature stratification. We restricted our analysis to wind directions of $\pm 60^{\circ}$ relative to the streamwise sonic axis (corresponding to easterly winds) in order to avoid flow distortion from the tower structure, and that ensured a fetch of $1500 \mathrm{~m}$ of flat snow. Drainage flows, waves and other typical features of stably stratified flows could be present in the measurements and we did not try to remove them from the measurements. Such features are expected to affect the dynamical stability of the flow, which we are quantifying through this analysis. Here we mostly focus on two quantities: the fluctuations of the vertical velocity $w^{*}$ and the angle $\alpha$ relative to the sonic axis. Both quantities are calculated on intervals of 6 $\mathrm{s}$, resulting in timeseries at the time step of $6 \mathrm{~s}$. This averaging removes some of the high frequency fluctuations but is kept short to ensure that non-turbulent fluctuations are not included in the averages (see discussion in 35. and [15]). The wind angle is calculated based on the 6 s-averaged horizontal wind components. It is used in order to have 
an indicator for horizontal motions. When motions are disorganized such as in presence of non-turbulent, meandering motions or submeso-motions, the fluctuations in the $6 \mathrm{~s}$ wind direction are expected to experience great excursions.

The dataset is separated in clusters as described in [12]. They were obtained by analysing the interactions between horizontal submeso scales motions and turbulence through the application of nonstationary multivariate autoregressive factor (VARX) models and finite-element clustering methods (FEM). The FEM-BV-VARX clustering procedure consists of representing the data (in this case the turbulent vertical velocity fluctuations) through a sequence of locally stationary statistical (VARX) models. The statistical models include the influence of external factors, which in this case are the horizontal submeso scales wind fluctuations. The clustering method of the turbulence data into different VARX processes is then done via a finite-element approach in which the most likely parameters of the different VARX processes are estimated simultaneously with the most likely sequence of switching among the clusters. The FEM algorithm computes the optimal clustering of the data through the computation of an optimal sequence of locally stationary fast VARX processes and a slow hidden process switching between them. In the end, each different set of VARX parameters corresponds to a different data cluster. This methodology was also used for analysis of the large-scale flow data: e.g., for identification of global atmospheric circulation regimes and blockings in the southern hemisphere [36, in the northern hemisphere [37] - as well as for the analysis of weather extremes 38. The review paper 39] contains a general overview of different non-stationary time series analysis methods for regime identification, together with many practical application examples. For further details about the method and its justification, reader is referred to [40] and its mathematical proofs especially the minimization problem to 41 .

Earlier analyses of the SnoHATS dataset highlighted the existence of four SBL flow regimes (clusters) [12. This number of clusters to be considered in the FEM-BV-VARX algorithm was selected invoking information criteria (AIC and BIC, see [12] for the detailed procedure) as suggested by Horenko [42]. Another possibility for identifying clusters is to use non-parametric methods of model identification [43. The four metastable regimes were analysed based on the interactions between horizontal non-turbulent motions on the so-called submeso scales (which can include wave-like motions, density currents or vortical structures) and turbulence. These differences in scale characteristics of the flow could induce different dynamical stability properties [13. The vertical velocity fluctuations were found to be related to horizontal submeso motions in two clusters (cluster 2 and 4 in what follows), whereas two clusters did not exhibit a strong relationship (cluster 1 and 3 in what follows). Moreover, one of the two submeso-influenced clusters, namely cluster 2, was found to exhibit a separation of scales between the turbulent and submeso scales, whereas scales were found to overlap in cluster 4 . We expect the forcing of turbulence by submeso motions to be related to dynamically unstable flows, and this is what we will quantify in the following analyses.

We use the $\Upsilon$ indicator on the vertical fluctuations $w^{*}$ and on the angle of the horizontal velocity $\alpha$ to discriminate between the stability of each cluster. An example of the time series analysed is shown in Figure 6. We apply our ARMA study on the four data collections with three different correlation windows $=\tau=5,10,15$ minutes as we want to investigate local and mesoscale effects on the stability. The $\Delta t$ is chosen to be 6 s. In fact, as shown in [12, below this time scale, the fluctuations consist mainly of isotropic turbulence. Since each cluster is made of several discontinuous time series, we analyse only those containing enough obervations to compute $\Upsilon$ for the $\tau \mathrm{s}$ considered.On Fig. 7 we report for the analysed sonic the logarithm of the empirical probability density function (EPDF) of $\Upsilon$ for the four clusters obtained as described before (left panels for $w^{*}$ and right panels for $\alpha$. We first remark that there are substantially no difference in the $\operatorname{EPDF} \Upsilon\left(w^{*}\right)$ for the same cluster but for different $\tau$ while the angle $\alpha$ is most sensitive to different time scale: for $\tau=5 \mathrm{~min}$, the values of $\Upsilon(\alpha)$ are larger than those obtained for $\tau=10,15$ minutes. For long time scales, this is an indication that $\alpha$ follows the direction of the average wind $\bar{\alpha}$ (induced by synoptic or mesoscale motions) and the dynamics mostly fluctuates around $\bar{\alpha}$ without extreme excursion. On the $\tau=5 \mathrm{~min}, \alpha$ is influenced by local turbulent or sub-meso motions which cause greater variations of the direction, reflected by the tendency of $\Upsilon$ in assuming larger values (see Marht et al. [44]).

We now analyse the differences between cluster. From the analysis of figure 7 , the most unstable ones appear to be the cluster 2 and 4 . These clusters are characterized by strong stratification and cluster 2 is further characterised by a separation of the turbulent and non-turbulent scales, whereas scales overlap in Cluster 4 . Within this cluster, the coherent motions propagates for finite time and produce high $\Upsilon$ values. The distribution of cluster 4 is squeezed toward smaller values of $\Upsilon$. It presents however few large $\Upsilon\left(w^{*}\right)$ values mostly for $\tau=15$ min, a signature of the broken equilibrium between turbulent and non-tubulent motions. Cluster 1 and 3 have a similar $\Upsilon$ distribution and they have been previously found to be obtained in weakly stratified PBL. The distribution of the total flux is very different in the periods extracted from clusters 2 and 4 [12. These dynamical stability results showing larger values 
Cluster 1
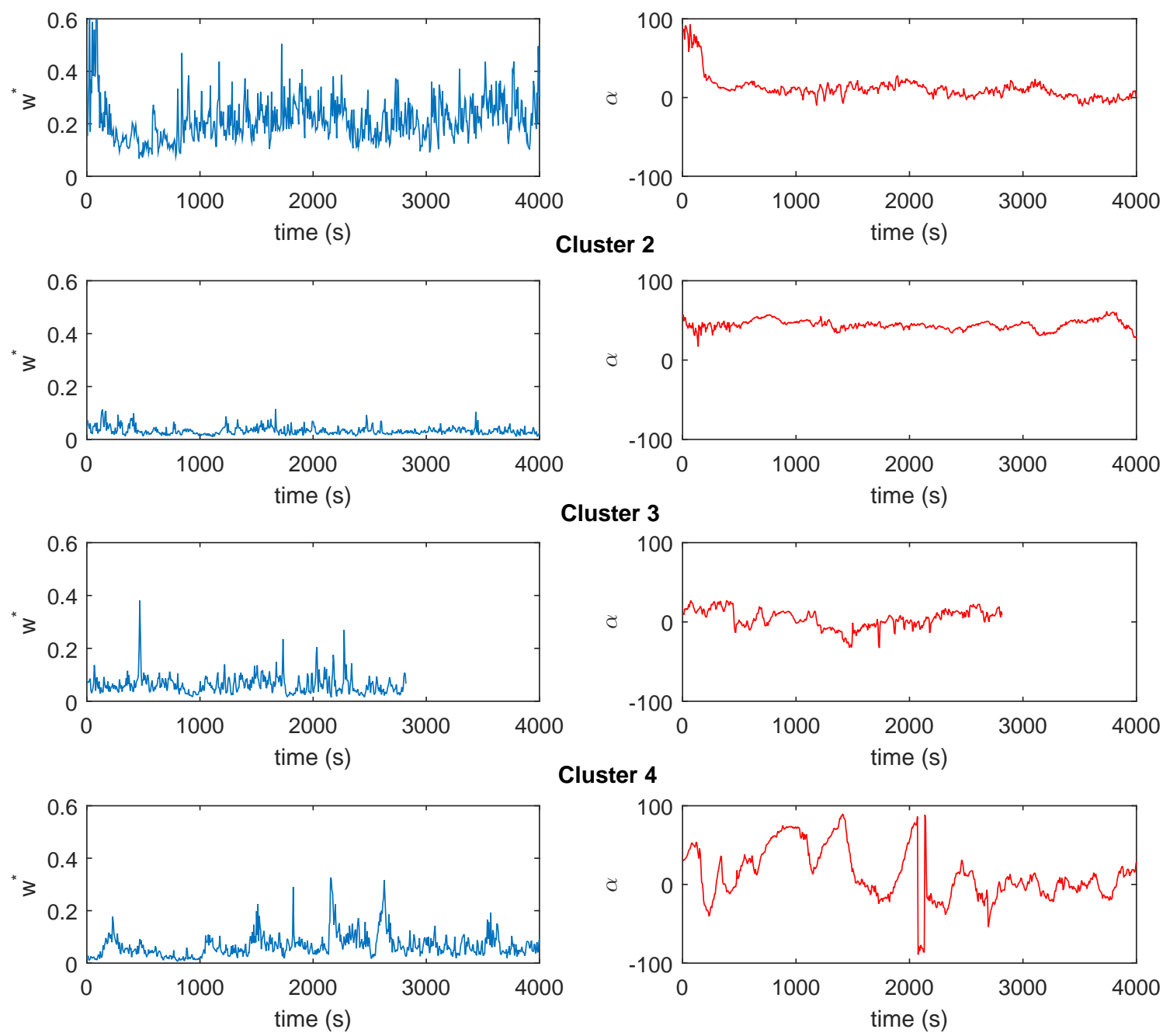

FIG. 6: Examples of signals analysed fo each cluster (from top to bottom). The wind vertical velocity fluctuations $w^{*}$ (left) and the angle $\alpha$ as a function of time.

of $\mathrm{Y}$ in the strongly stable scenarios and smaller values in the weakly stable scenarios are also in agreement with the weakly stable flow observations falling onto the stable branch of the idealized Couette flow model, as discussed in the introduction.

\section{DISCUSSION}

We have built an indicator $\Upsilon$ as that is able to quantify the a dynamical stability of metastable states or fixed points. We have applied it in various systems of increasing complexity, ranging from a simple conceptual model (the Lorenz 17. attractor), through laboratory turbulent flows. to atmospheric wind measurements. The $\Upsilon$ indicator correctly detects the change in the stability of metastable states regardless to the complexity of the underlying system. The main advantages of using $\Upsilon$ with respect to the computation of other dynamical systems based techniques (e.g. the Lyapunov exponents), is the local nature of this indicator and the extremely low requirements in terms of data. Here, by local we mean around a metastable states identified in the phase space, as we have illustrated in the example of the Lorenz and the von Karman swirling flow attractors. The computation of Lyapunov exponents 45] requires long time series and a complex orthogonalization procedure, $\Upsilon$ a maximun likelihood fit only. In the context of PBL 

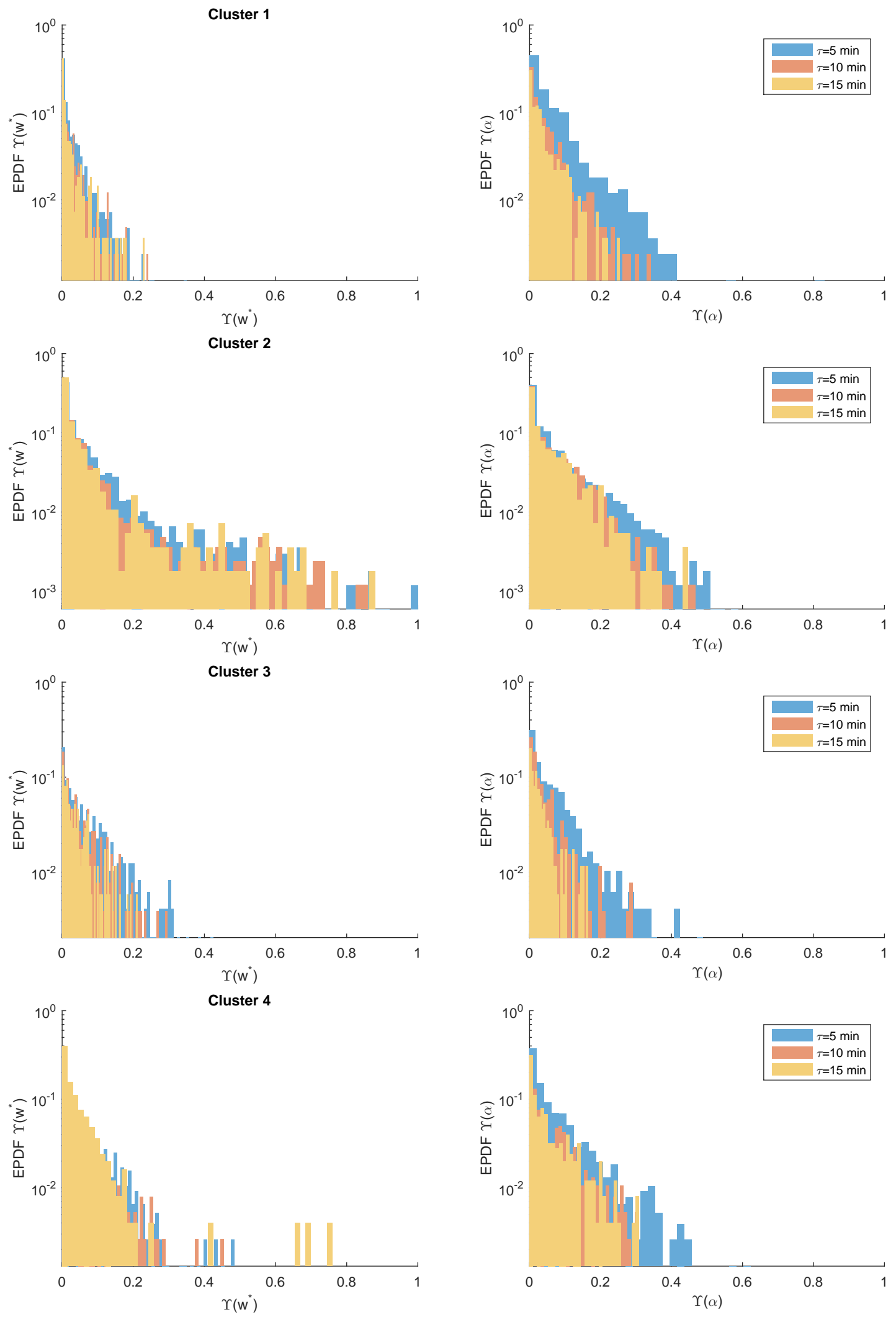

FIG. 7: ARMA study on the 4 different clusters (from top to bottom) of sonic 6 for the fluctuations of the vertical velocity $w^{*}$ (left) and for the wind direction $\alpha$ (right). Different colors correspond to different $\tau$, as in the legend. 
parametrization, the information extracted via $\Upsilon$ can be used to build better stochastic models of the PBL. Since higher $\Upsilon$ values imply the need of more complex stochastic models, high PBL stratifications (Cluster 2 and 4 in the analysed datasets) should be modeled with higher order stochastic processes. On the other hand, the exponential distribution of $\Upsilon$ fo weakly stable PBL, suggest that a simple $\operatorname{AR}(1)$ model is suitable to represent the dynamics. We will further pursue the research in this direction and build statistical models which are capable to reproduce the exponential (weakly stable PBL) or power law (highly stable PBL) of $\Upsilon$ e.g. by introducing a stochastic behavior of the autoregressive and/or moving average coefficients.

\section{ACKNOWLEDGMENTS}

Illia Horenko and the anonymous reviewers are gratefully acknowledged for their careful comments. NV received support from the Deutsche Forschungsgemeinschaft (DFG) through grant number VE 933/2- 1.

* Electronic address: Correspondence to: davide.faranda@cea.fr

[1] S. Bony and J.-L. Dufresne, Geophysical Research Letters 32 (2005).

[2] E. McGrath-Spangler, A. Molod, L. Ott, and S. Pawson, Atmos. Chem. Phys 15, 7269 (2015).

[3] G. Svensson and J. Lindvall, Journal of Climate 28, 5233 (2015).

[4] I. Sandu, A. C. M. Beljaars, P. Bechtold, T. Mauritsen, and G. Balsamo, Journal of Advances in Modeling Earth Systems 5, $117(2013)$.

[5] A. Holtslag, M. Tjernström, S. Basu, A. C. M. Beljaars, G. Svensson, P. Baas, B. Beare, F. C. Bosveld, J. Cuxart, J. Lindvall, et al., Bulletin of the American Meteorological Society 94, 1691 (2013).

[6] B. J. H. van de Wiel, A. F. Moene, R. J. Ronda, H. De Bruin, and A. Holtslag, Journal of Atmospheric Sciences 59, 2567 (2002).

[7] J. Sun, L. Mahrt, and R. M. Banta, Journal of the Atmospheric Sciences (2012).

[8] J. Sun, L. Mahrt, C. J. Nappo, and D. H. Lenschow, Journal of Atmospheric Sciences 72, 1484 (2015).

[9] Y. Kang, D. Belušić, and K. S. Miles, Quarterly Journal of the Royal Meteorological Society (2015).

[10] L. Mahrt, Annual Review of Fluid Mechanics 46, 23 (2014).

[11] D. Cava, L. Mortarini, U. Giostra, R. Richiardone, and D. Anfossi, Quarterly Journal of the Royal Meteorological Society 143, 661 (2016).

[12] N. Vercauteren and R. Klein, Journal of the Atmospheric Sciences 72, 1504 (2015).

[13] L. Mahrt and C. K. Thomas, Boundary-Layer Meteorology pp. 1-19 (2015).

[14] A. H. Monahan, T. Rees, Y. He, and N. McFarlane, Journal of Atmospheric Sciences 72, 3178 (2015).

[15] N. Vercauteren, L. Mahrt, and R. Klein, Quarterly Journal of the Royal Meteorological Society 142, 2424 (2016).

[16] B. J. H. van de Wiel, A. F. Moene, G. J. Steeneveld, O. K. Hartogensis, and A. Holtslag, Flow, Turbulence and Combustion 79, 251 (2007).

[17] E. N. Lorenz, Journal of the atmospheric sciences 20, 130 (1963).

[18] E. Bou-Zeid, C. Higgins, H. Huwald, C. Meneveau, and M. B. Parlange, Journal of Fluid Mechanics 665, 480 (2010).

[19] S.-H. Poon and C. W. Granger, Journal of economic literature 41, 478 (2003).

[20] H. Akaike, Applied Time Series Analysis pp. 1-23 (1978).

[21] H. Akaike, in Selected Papers of Hirotugu Akaike (Springer, 1998), pp. 199-213.

[22] D. Faranda, F. M. E. Pons, E. Giachino, S. Vaienti, and B. Dubrulle, Communications in Nonlinear Science and Numerical Simulation 29, 233 (2015).

[23] D. Faranda and D. Defrance, Earth System Dynamics 7, 517 (2016), URL http://www.earth-syst-dynam.net/7/517/ $2016 /$

[24] A. Uchida, K. Yoshimura, P. Davis, S. Yoshimori, and R. Roy, Physical Review E 78, 036203 (2008).

[25] D. Faranda, G. Messori, and P. Yiou, Scientific reports 7, 41278 (2017).

[26] H. Fujisaka, Progress of theoretical physics 70, 1264 (1983).

[27] L. Arnold, W. Kliemann, and E. Oeljeklaus, in Lyapunov exponents (Springer, 1986), pp. 85-125.

[28] P.-P. Cortet, A. Chiffaudel, F. Daviaud, and B. Dubrulle, Physical Review Letters 105, 214501 (2010).

[29] B. Saint-Michel, B. Dubrulle, F. Ravelet, and F. Daviaud, Physical Review Letters 111, 234502 (2013).

[30] D. Faranda, Y. Sato, B. Saint-Michel, C. Wiertel, V. Padilla, B. Dubrulle, and F. Daviaud, Physical Review Letters 119, $00000(2017)$

[31] S. Thalabard, B. Saint-Michel, E. Herbert, F. Daviaud, and B. Dubrulle, New Journal of Physics 17, 063006 (2015).

[32] N. H. Packard, J. P. Crutchfield, J. D. Farmer, and R. S. Shaw, Physical Review Letters 45, 712 (1980).

[33] T. Sauer, J. A. Yorke, and M. Casdagli, Journal of statistical Physics 65, 579 (1991).

[34] B. Saint-Michel, F. Daviaud, and B. Dubrulle, New Journal of Physics 16, 013055 (2014).

[35] L. Mahrt, S. J. Richardson, N. Seaman, and D. Stauffer, Quarterly Journal of the Royal Meteorological Society 138, 1430 (2012). 
[36] T. J. O'kane, J. S. Risbey, D. P. Monselesan, I. Horenko, L. Christian, and E. Franzke, Climate dynamics 46, 3567 (2016).

[37] J. S. Risbey, T. J. O'Kane, D. P. Monselesan, C. Franzke, and I. Horenko, Journal of the Atmospheric Sciences 72, 35 (2015).

[38] O. Kaiser, D. Igdalov, and I. Horenko, Multiscale Modeling \& Simulation 13, 594 (2015).

[39] P. Metzner, L. Putzig, and I. Horenko, Communications in Applied Mathematics and Computational Science 7, 175 (2012).

[40] I. Horenko, Dynamics of Atmospheres and Oceans 49, 164 (2010).

[41] I. Horenko, Journal of the Atmospheric Sciences 67, 1559 (2010).

[42] K. P. Burnham and D. R. Anderson, Model selection and multimodel inference: a practical information-theoretic approach (Springer Science \& Business Media, 2003).

[43] S. Gerber and I. Horenko, Science advances 1, e1500163 (2015).

[44] L. Mahrt, Boundary-Layer Meteorology 88, 255 (1998).

[45] A. Wolf, J. B. Swift, H. L. Swinney, and J. A. Vastano, Physica D: Nonlinear Phenomena 16, 285 (1985). 